\title{
True directed forgetting in pigeons may occur only when alternative working memory is required on forget-cue trials
}

\author{
KAREN L. ROPER, DAREN H. KAISER, and THOMAS R. ZENTALL \\ University of Kentucky, Lexington, Kentucky
}

\begin{abstract}
Results of directed-forgetting research with pigeons are difficult to interpret because of alternative nonmemorial accounts of performance decrements and important procedural differences from comparable research with humans. Prior research has noted the absence of directed forgetting when artifacts have been removed (e.g., nonreward following forget cues and differences in response patterns on remember and forget trials in training). In this article, it is argued that, in human directed-forgetting research, presentation of a forget cue allows for the reallocation of memory maintenance to items to be remembered. In the present experiment, true directed forgetting is found when nonmemorial performance decrements are eliminated and forget cues allow for the reallocation of sample memory to test-relevant cues.
\end{abstract}

Evidence from human memory research indicates that humans can exert active control over the maintenance of memories. In studies involving "directed-forgetting" procedures, in which presentation of a list of items includes signals to remember some of those items but not others, humans show better memory for to-be-remembered items than for items that are signaled to be forgotten (e.g., Bjork, 1972; Epstein, 1972). A possibly related phenomenon has been reported in pigeons using a variation on a task that has been used extensively in research on animal memory, delayed matching-to-sample (DMTS; see Blough, 1959). This task typically involves training pigeons to peck a sample stimulus (e.g., a red or a green hue) and then to choose, after a delay, between two comparison stimuli (e.g., red and green). A response to the stimulus that matches the sample is reinforced. The finding that pigeons perform less accurately as the delay interval between the offset of the sample and onset of the comparisons is lengthened (e.g., Blough, 1959) suggests that pigeons forget the identity of the sample stimulus over time. More recently, a version of DMTS has been adapted to study directed forgetting in pigeons (e.g., Grant, 1981; Maki \& Hegvik, 1980; Maki, Olson, \& Rego, 1981; Santi \& Savich, 1985 ) and other nonverbal species (Grant, 1982; Roberts, Mazmanian, \& Kraemer,1984). In this task, a conditional cue is presented during the delay between sample and comparisons indicating whether or not memory for the

Preparation of this paper was supported by National Science Foundation Grant BNS 9019080 and National Institute of Mental Health Grant 1 R01 MH45979 to the third author. We thank Lou M. Sherburne for her assistance. Correspondence concerning this article should be addressed to K. L. Roper, who is now at Department of Psychology, University of Wisconsin, Eau Claire, WI 54702.

-Accepted by previous editor, Vincent M. LoLordo sample will be tested on that trial. Following the remember $(R)$ cue, the trial terminates in the standard two-choice comparison test. Following the forget $(\mathrm{F})$ cue, memory for the sample is not tested (e.g., the trial ends without presentation of comparisons). After substantial experience with the $R$ and $F$ cues, one can assess memory on F-cue trials by presenting infrequent "probe" trials involving the presentation of comparison stimuli following the $F$ cue. Poor performance on F-cue-probe trials has been taken as evidence that animals can be "directed to forget" the sample stimulus or, more generally, that animals show a kind of flexibility in the maintenance of memory that is similar to that of humans.

In a review of the animal directed-forgetting literature, Roper and Zentall (1993) noted that the effectiveness of $F$ cues in producing a consistent decrement in DMTS probetrial performance seems to depend on the use of the "omission" procedure. With this procedure, the $\mathrm{F}$ cue, which signals the absence of a memory test, is followed immediately by the intertrial interval (ITI). Possible sources of F-cue disruption in the omission procedure that are not attributable to memory loss include (1) frustration produced by the nonoccurrence of reward following the F cue, (2) inattention to the comparison stimuli following F-cue presentation, and (3) a generalization decrement (or surprise) when $\mathrm{F}$ cues are followed by memory test on probe trials. Any of these factors could produce a decrement in probetrial performance that is not directly attributable to the $\mathrm{F}$ cue's effect on memory. In support of this hypothesis, the presence of a directed-forgetting effect appears to depend on events that follow the F cue during training. For example, when a "substitution" procedure has been used, in which the comparison stimuli that normally follow the $F$ cue are replaced by one of a number of (sample-independent) events, including reinforcement, it has often been reported that there is little disruption of performance on F-cue- 
probe trials (e.g., Kendrick, Rilling, \& Stonebraker, 1981; Maki \& Hegvik, 1980; Maki et al., 1981).

In those experiments involving substitution procedures in which directed forgetting has been reported, Roper and Zentall (1993) proposed that, on F-cue-probe trials, the pattern of responding required following the $F$ cue was different from what was established following the $F$ cue during training. According to this notion of response compatibility, the end-of-trial behavior pattern on F-cue trials (relative to that on R-cue trials) determines whether or not performance will be disrupted on the unexpected memory test that occurs on F-cue-probe trials. In general, when the pattern of responding is the same following $R$ and $F$ cues (e.g., a two-alternative forced choice involving a single response with reinforcement possible), directed forgetting does not occur. However, if post-F-cue responding either is not required for reinforcement or is reinforced indiscriminately, there is typically a disruption of performance on F-cue-probe trials comparable to that shown with the omission procedure. Thus, the performance decrement often seen on F-cue-probe trials in directed-forgetting research may be produced by nonmemorial factors.

Alternatively, it has been proposed that the failure to find disrupted matching accuracy on F-cue-probe trials when substitution procedures have been used occurs because the $\mathrm{F}$ cue does not terminate memory search (Kendrick \& Rilling, 1986). Memory search is not terminated, according to Kendrick and Rilling, because a response decision (i.e., which side key to peck) cannot be made until the simple simultaneous discrimination is presented.

Regardless of the reason for the high level of performance on F-cue -probe trials with substitution procedures, the finding that there is no deficit in F-cue-probe-trial performance when response-compatible substitution procedures are used makes it highly unlikely that the disrupted performance often found in directed-forgetting research results merely from the change in stimulus conditions between training and test. In other words, the high level of performance on test trials following substitution training suggests that there is no generalization decrement associated with unexpected comparisons on F-cue-probe trials. Although there is some suggestion in the literature that, in a conditional discrimination in which each sample maps onto more than one comparison (i.e., a one-to-many matching task), signaling which comparison will appear can facilitate matching accuracy (Grant \& MacDonald, 1990). Such an effect does not appear to be present in directedforgetting preparations involving substitution procedures.

The failure to find a directed-forgetting effect when compatible-response-pattern substitution procedures have been used does not mean that animals are incapable of utilizing $R$ and $F$ cues to determine what is maintained in memory. Instead, it suggests that the tasks that have been used to study directed forgetting in animals may not have adequately modeled directed-forgetting tasks used with humans. With human subjects, items are given in list form, with cues designating which items are to be remembered and which are to be forgotten. Because the list procedure involves both remember and forget items within the same trial, humans may choose to use the interval between list items to rehearse previous remember items. Rehearsal of previous remember items would be especially likely during presentation of an $F$ cue. Thus, a reduction in the processing of one type of item (F-cued) can allow for the increased processing of others (R-cued items).

On the other hand, when directed forgetting is assessed in animals using a matching task, they are generally trained with only one item per trial. A small number of directedforgetting experiments with pigeons have involved a multiple-item memory task (Grant, 1984, 1986, 1989). In these experiments, two successively presented samples were each followed by an R or an F cue. Grant (1984) trained pigeons on a standard directed-forgetting task with $R$ and $F$ cues and tested them with double-sample probe trials. Of particular interest was the finding that, when an $R$ cue followed the second sample, accuracy was higher when the first sample was followed by an $F$ cue than when it was followed by an $R$ cue. Thus, the $F$ cue served to reduce the interfering effects of the first sample on the second. The problem with introducing double samples on test trials is that it may produce an ambiguity of instructions (i.e., it is not clear which is the "true" sample). To remedy this ambiguity, Grant (1986) trained pigeons on a directedforgetting task involving double-sample trials with an $F$ cue following the first sample and an $\mathrm{R}$ cue following the second. The disrupted accuracy on probe trials in which the $R$ cue followed both samples again suggested that those cues can influence the interfering effects of the first sample. But, as Grant (1989) noted, although it is assumed that the $\mathrm{F}$ cue presented between the two samples retroactively affected the first sample, it may have also proactively affected the way the second sample was processed. Furthermore, it is not clear that Grant's (1989) use of single-sample probe trials avoids the problems inherent in the doublesample procedure (see Roper \& Zentall, 1993). Whenever two samples representing conflicting instructions are presented on a trial, there is a reasonable likelihood that an instructional deficit will occur on probe trials.

The purpose of this research was to provide pigeons with a directed-forgetting task that is more analogous to the procedures used to assess human memory (i.e., procedures in which $\mathrm{F}$ cues allow for the reallocation of memory to R-cue items). The present research also involved controls for possible procedural artifacts common in directedforgetting research (Roper \& Zentall, 1993). In the present study, pigeons were trained on standard R-cue DMTS trials with a test of sample memory following the R cue. Fcue training, however, involved two functionally different $F$ cues (one per trial) followed by sample-irrelevant comparisons, with correct responding contingent on the identity of the F cue. Thus, the F cues also served as samples for a conditional discrimination embedded in the matching task. This F-cue procedure resembles standard substitution procedures in that (1) reward is available for correct comparison choice on F-cue trials, and (2) a response to one of the side keys is required to obtain reinforcement. The procedure also ensures that a similar pattern of behavior occurs on both $\mathrm{R}$-and $\mathrm{F}$-cue trials in training. What 
makes this design more similar to that used with humans is that presentation of an F cue signals not only that memory for the sample will not be tested but also that memory for a different stimulus (in this case, the $F$ cue itself) will be tested. This procedure thus provides the animal with an incentive to reallocate memory maintenance from the sample to the $F$ cue. Without such reallocation during training, the maintenance of sample memory might be expected to interfere with F-cue-trial performance. If this reallocation is successful, one would expect to find a decrement in performance when sample memory is tested on F-cue-probe trials. Furthermore, if this analysis of directed-forgetting research with animals is correct, the performance decrement should be found in spite of the similar patterns of reinforced responding on $\mathrm{R}$ - and $\mathrm{F}$-cue trials in training.

The present design may also allow for assessment of acquisition of the $R$ and $F$ cues as instructions during training. Because delayed matching is trained prior to the introduction of $R$ and $F$ cues, it is expected that accuracy on $\mathrm{R}$-cue trials will be high initially. As $\mathrm{F}$ cues begin to gain control over comparison choice, however, one might expect to see some drop in performance on R-cue trials due to a shift in attention from samples to delay cues.

\section{METHOD}

\section{Subjects}

The subjects were 6 White Carneaux pigeons purchased from Palmetto Pigeon Plant (Sumter, SC). All birds had served in previous experiments that involved matching-to-sample. The birds were kept at $80 \%$ of their free-feeding body weights on a diet of Purina Pigeon Grains provided primarily during experimental sessions. The birds were housed in individual cages in a temperature-controlled colony room that was illuminated on a 12:12-h light:dark schedule. Water and grit were freely available in the home cages.

\section{Apparatus}

The birds were tested in a BRS/LVE (Beltsville, MD) operant chamber measuring $37.5 \mathrm{~cm}$ high, $31 \mathrm{~cm}$ from front panel to back wall, and $35.5 \mathrm{~cm}$ from side to side. Five horizontally aligned rectangular $(3.5 \times 3 \mathrm{~cm})$ response keys were located on the front panel $1 \mathrm{~cm}$ apart and $26.5 \mathrm{~cm}$ from the center of the key to the floor of the chamber. Only the center three keys were used in the experiment. An inline projector was mounted behind each of the three response keys. The center projector could illuminate green, red, and blue fields (Kodak Wratten Filter Nos. 60, 26, and 38, respectively), and a solid white field (no filter). Also in the center projector were a dot $(5 \mathrm{~mm}$ diameter) and an annulus ( $16 \mathrm{~mm}$ outside diameter, $13 \mathrm{~mm}$ inside diameter), both white on a black background and centered on the display. Red and green fields (identical to that of the center projector) as well as vertical and horizontal lines (consisting of three white lines $2.4 \mathrm{~cm}$ long, $0.3 \mathrm{~cm}$ wide, and $0.3 \mathrm{~cm}$ apart) could be illuminated from the two side projectors. The bottom edge of a centrally located feeder aperture $(6 \times 5.5 \mathrm{~cm})$ was situated $11.5 \mathrm{~cm}$ from the wire-mesh floor. White noise (at $72 \mathrm{~dB}$ ) and the sound of an externally mounted exhaust fan masked extraneous sounds. The chamber was controlled by a microcomputer located in an adjacent room.

\section{Procedure}

Because of their training in previous experiments, all birds were accustomed to the operant chamber and had acquired the keypeck response prior to the start of the experiment. Thus, training on the matching task began immediately.
Acquisition of DMTS. Each trial began with the presentation of a red or a green sample on the center key. The pigeons were required to peck the sample 10 times (FR 10), after which the center key was turned off and the two side keys were immediately illuminated with red and green comparison stimuli. A single peck to the matching comparison stimulus resulted in reinforcement, terminated the stimulus display, and started a $10-\mathrm{sec}$ ITI. A single peck to the nonmatching comparison stimulus terminated the stimulus display and initiated the ITI. Training with this task continued until each bird reached an individual criterion of $90 \%$ correct or better for two consecutive sessions. On the following day, delay training began. The delay interval was initially set at $1 \mathrm{sec}$. As each bird reached criterion, the delay was incremented to $2 \mathrm{sec}$ and then $4 \mathrm{sec}$. Each session consisted of 96 trials, and each color served as the sample on 48 randomly assigned trials. Position of the correct side key (left or right) was balanced for trials involving each sample color, and no more than three consecutive trials involved the same sample color or correct comparison position. Each pigeon received five additional training sessions after reaching criterion at the 4-sec delay.

Training with delay-interval cues. Following delayed matching training, a cue (dot, circle, blue, or white) was introduced on the center key during the delay interval on each trial. The cue remained on throughout the delay. For half the birds, dot and circle served as $\mathrm{R}$ cues and blue and white were $F$ cues. Two $R$ cues were used to control for number of $F$ cues, but the $R$ cues were not correlated with either the sample or the location of the correct comparison. The stimuli used as $\mathrm{R}$ and $\mathrm{F}$ cues were reversed for the remaining birds. Presentation of the dot, circle, blue, or white stimuli occurred equally often. Trials were arranged in random order, with the stipulation that no cue was presented on more than three consecutive trials. Red and green comparison stimuli immediately followed the delay on R-cue trials. On those trials, pecks to the comparison that matched the initial sample were reinforced. Vertical and horizontal lines served as comparisons on all F-cue trials. Reinforcement for choice of vertical or horizontal was contingent on the identity of the F cue. Responses to vertical were reinforced following one of the two $F$ cues, and responses to horizontal were reinforced following the other $F$ cue. Thus, the F cues served as samples in a new zero-delay matching task. Each pigeon was required to reach a criterion of $90 \%$ correct performance for two consecutive sessions on both $\mathrm{R}$-cue and $\mathrm{F}$ cue trials before probe trials were introduced. For pigeons that had difficulty attaining criterion after the insertion of delay cues, the number of pecks required to the sample was increased from 10 to 20 after a minimum of 50 sessions of training with the delay cues. For the birds that still were unable to reattain a high level of DMTS accuracy, the number of trials involving $R$ and $F$ cues was changed to 72 and 24, respectively.

Probe sessions. Probe sessions were identical to delay-cue sessions with the exception that four probe trials were added to each session. One probe trial was added to each block of 24 trials. Each of the probe trials involved presentation of one of the two $\mathrm{F}$ cues, followed by red and green comparison stimuli and reinforcement for choice of the comparison that matched the sample. Each of the $F$ cues was presented on two probe trials in each probe session. Sample color was equally often red and green, and position of the correct comparison was balanced across probe trials. Testing with probe trials continued for 48 sessions. The design of the experiment is presented in Figure 1.

\section{RESULTS}

From the start of training, the pigeons took an average of 47.2 sessions to reach criterion at the 4-sec delay. When delay cues were introduced, matching accuracy on sampletested (R-cue) trials showed an initial drop of $15 \%$ correct on the first delay-cue session. Of greater interest was the tendency of the birds to continue to drop in performance on 

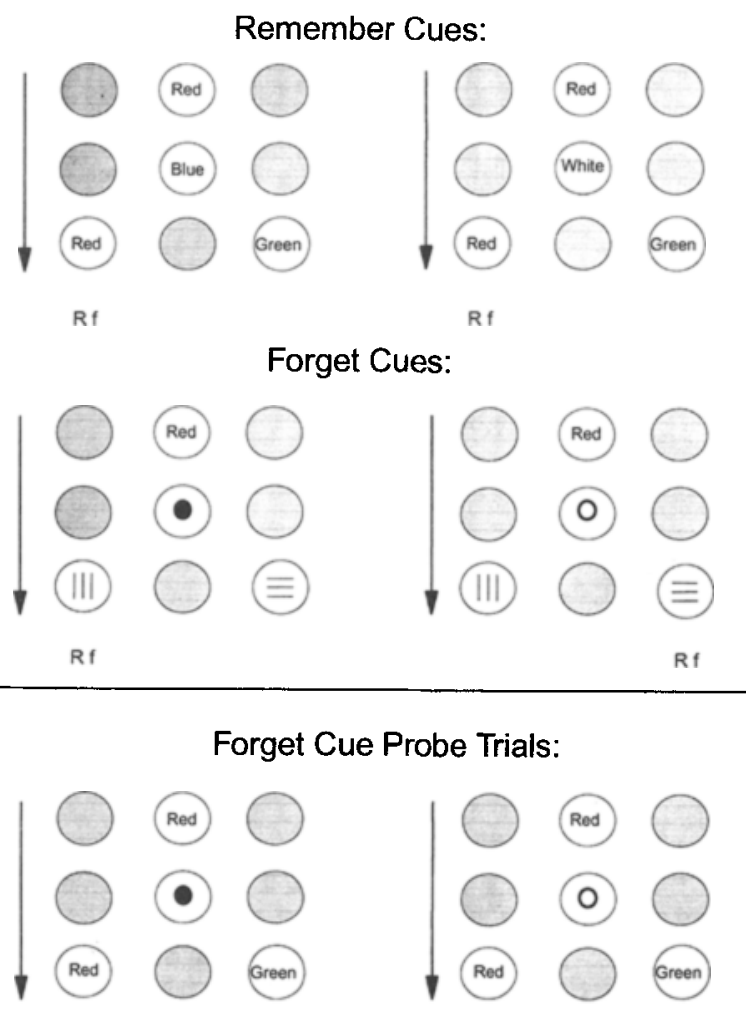

Rf

Figure 1. Directed-forgetting design with remember cues (blue and white) followed by comparison test relevant to the initial sample, and forget cues (dot and circle) followed by vertical and horizontal lines (remember and forget cues were reversed for half of the pigeons). Sample-matching responses were reinforced following remember cues. Responses to vertical and horizontal comparisons were reinforced depending on the identity of the preceding forget cue.

R-cue trials as they learned the F-cue matching task. Only one bird, S2, failed to show this pattern, but this bird learned the task at an exceptionally fast rate. Performance for each of the 6 birds on R- and F-cue trials during delay-cue training is displayed in Figure 2. For most pigeons, acquisition of the conditional discrimination involving the $F$ cues was rapid, and the remaining training sessions were required to reattain a high level of performance on R-cue trials.

The reacquisition of $R$-cue-trial performance from the present study was compared with data collected from an experiment in which a standard substitution procedure involving a simple simultaneous discrimination (Zentall, Roper, \& Sherburne, 1995) was used. This comparison is useful because both experiments used the same samples, comparisons, and substituted comparison stimuli; however, Zentall et al.'s F-cue task did not involve a conditional discrimination, whereas our F-cue task did. Pigeons trained with the standard substitution procedure took an average of 28.0 sessions to reacquire R-cue-trial performance (to a criterion of two consecutive sessions at $80 \%$ correct or better), whereas the pigeons in the present ex- periment took an average of 58.5 sessions to reacquire Rcue-trial performance to a comparable criterion.

The probe-session data were pooled over the 48 probe sessions. Mean performance on R-cue and F-cue-probe trials, as well as matching performance on the standard F-cue trials, is shown in Figure 3. Mean performance on R-cue trials ( $86.4 \%$ correct) was significantly higher than that on $\mathrm{F}$-cueprobe trials $(73.0 \%$ correct $)[F(1,5)=13.79]$. Probe-session performance for each bird is also presented in Figure 3.

Finally, an analysis was performed to assess the relation between the disruption of R-cue-trial performance in delay-cue training and the disruption of performance on F-cue-probe trials in test. A correlation analysis revealed that there was a positive relation between the number of days taken to learn the task in the training phase and the degree of probe-trial disruption. The Pearson product moment correlation ( $r=.66$ ) was only marginally significant, however, perhaps because of the lack of statistical power.

\section{DISCUSSION}

The purpose of this experiment was to test for the presence of directed forgetting in pigeons under conditions that (1) controlled for the motivational, attentional, and behavioral effects of F-cue training on DMTS performance and (2) more closely paralleled conditions used in human memory research. In keeping with human directedforgetting procedures, the F-cue test procedure in this experiment allowed for a more list-like presentation of memory items (e.g., F cues allow for reallocation of memory maintenance to $R$ items).

All 6 pigeons in this experiment showed reduced matching accuracy on F-cue-probe trials relative to R-cue trials. This result indicates that evidence for directed forgetting can be found in the absence of performance deficits resulting from nonmemorial factors, such as the unavailability of reinforcement or differences in response compatibility between F-cue trials in training versus F-cue-probe trials in test. Furthermore, the fact that the disruption of matching performance does not typically occur when compatibleresponse substitution procedures are used (e.g., Kendrick et al., 1981; Maki \& Hegvik, 1980; Maki et al., 1981) suggests that the present effects cannot be attributed to the novelty (or generalization decrement) associated with F-cueprobe trials.

Examination of the pattern of performance on R- and Fcue trials during delay-cue training (see Figure 2) suggests that there may be a tradeoff between correct matching to the samples (R-cue trials) and correct matching to the delay cues (F-cue trials). Thus, the birds that had difficulty maintaining performance on R-cue trials while learning the F-cue task may have been selectively attending to the delay cues at the expense of the samples during acquisition of the F-cue task. Comparison of performance on delayed-matching trials (R-cued) during delay-cue training and differences in performance between $\mathrm{R}$-cue trials and F-cue-probe trials in test suggests support for this reallocation hypothesis. The pigeons (S2 and S4) that learned to deal with the R- and F-cue tasks quickly during training may have been able to maintain 


\section{-Forget $\quad$ Remember}

S1

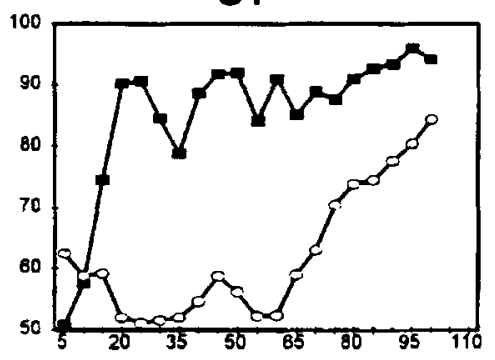

S3

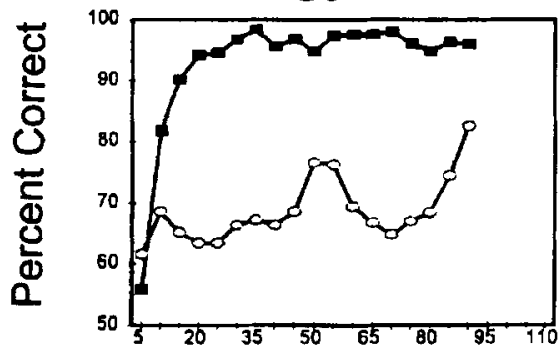

S5

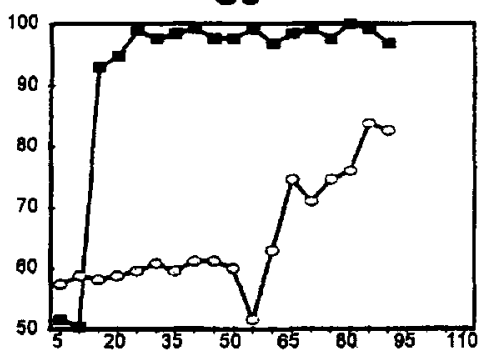

S2

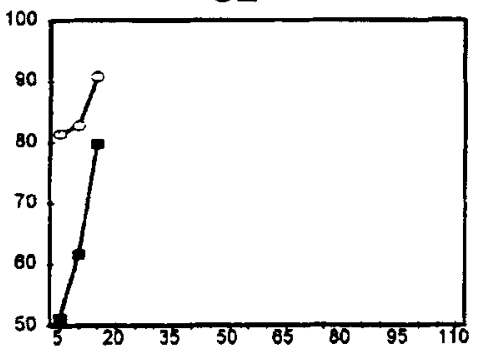

54

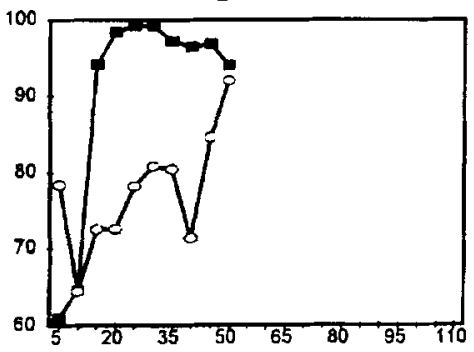

S6

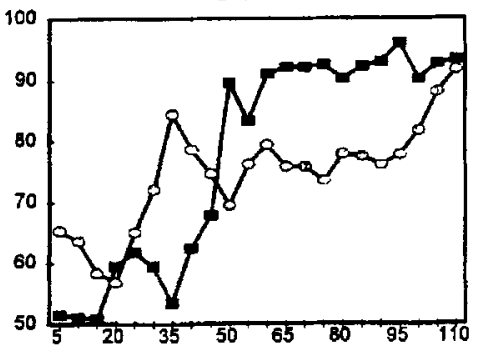

Training Sessions (Blocks of 5)

Figure 2. Individual and mean performance on remember- and forget-cue trials in blocks of five training sessions.

both the sample and the $\mathrm{F}$ cue in memory at the same time and, thus, showed little performance loss on F-cue -probe trials in test (S3 was an exception). On the other hand, the pigeons ( $\mathrm{S} 1, \mathrm{~S} 5$, and $\mathrm{S} 6$ ) that showed the greatest drop in accuracy on F-cue-probe trials also took many sessions to recover a high level of matching accuracy on R-cue trials.

In addition, the present results fail to support Kendrick and Rilling's (1986) account of the typical failure to find directed-forgetting effects when substitution procedures have been used (see Kendrick et al., 1981; Maki \& Hegvik, 1980; Maki et al., 1981; Zentall et al., 1995). According to Kendrick and Rilling, substitution procedures are generally sufficient to maintain rehearsal of the sample, whereas omission procedures are not. In the present research, although the memory demands of the substituted task were even greater than those of typical substitution procedures, they were not sufficient to maintain the level of memory for the sample found on control trials.
The present results are also relevant to a related hypothesis, suggested by a reviewer, that, in the typical directedforgetting research with substitution procedures, the "instructions" are confusing. In other words, in the case of omission procedures, it may be relatively easy to distinguish between instructions to remember the sample versus remember nothing, whereas, with substitution procedures, it may be harder to distinguish between instructions to remember the sample and those to prepare to perform the F-cue-signaled discrimination. Under such conditions, it may be easier for the pigeon to continue remembering the sample. The results of the present study suggest, however, that instructional confusion during training is not likely to account for the failure to find a directed-forgetting effect when substitution procedures are used, because the procedures used in the present experiment should have produced at least as much confusion as that in the typical substitution procedure. Nevertheless, strong evidence for directed forgetting was still found. 


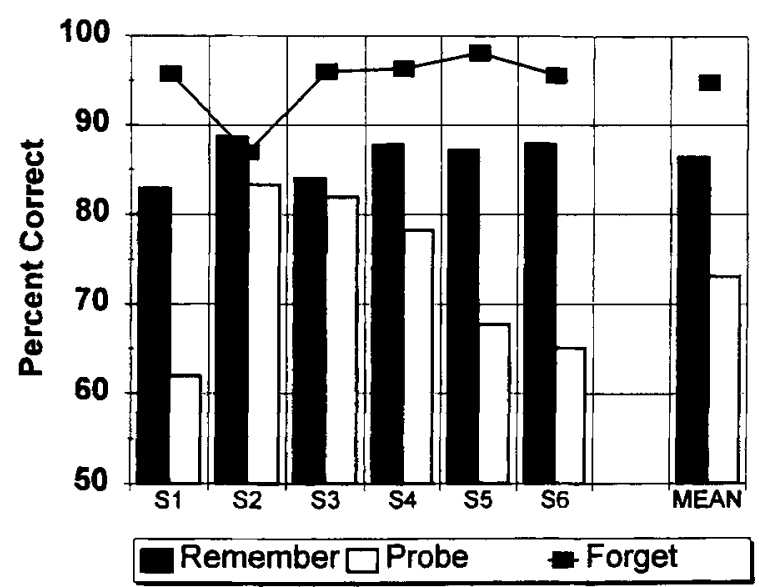

Figure 3. Individual and mean test performance on remembercue, forget-cue, and forget-cue-probe trials.

The failure to find disrupted performance on F-cueprobe trials when typical substitution procedures are used (e.g., Kendrick et al., 1981; Maki \& Hegvik, 1980; Maki et al., 1981; Zentall et al., 1995) also suggests that it is unlikely that the generalization decrement between training and test can account for the decrement in performance on F-cue-probe trials in the present experiment. This conclusion is particularly clear if one compares the results of the present experiment with data reported by Zentall et al., using a typical substitution procedure. In both experiments, red and green hues presented as comparisons on $R$-cue trials were replaced by vertical and horizontal lines on F-cue-probe trials, yet Zentall et al. found no evidence of disrupted matching accuracy in this group.

Finally, an interesting finding from the human directedforgetting literature is that although F-cue items are generally less well remembered than are R-cue items, R-cue items are generally better remembered than they would have been in the absence of F-cue items (i.e., for subjects who are asked to remember all of the items; see, e.g., Epstein, 1972). Thus, it is suggested that the resources that would have been allocated to the F-cue item are, at least in part, reallocated to the R-cue (i.e., remaining) items. It may be possible to demonstrate a similar facilitation of $\mathrm{R}$ items (relative to controls) with procedures similar to those used here. If, for example, control pigeons were trained on a task in which $R$ cues were always followed by a test of sample memory, whereas $F$ cues were sometimes followed by a test of memory for the F cue (as in the present experiment) and at other times followed by a test of sample memory, pigeons would be unable to reallocate resources on F-cue trials. If this were the case, control pigeons should have a more difficult time acquiring a high level of R-cue matching performance than did the pigeons in the present study. Such a demonstration would provide additional evidence for the similarity in mechanism between directed forgetting in humans and pigeons.

The results of this experiment suggest that, when memory demands comparable to those made on humans are made on animals, animals show performance deficits on probe-trial tests that are similar to the directed-forgetting effects shown by humans. The advantage of an approach that borrows techniques used in the study of human cognition (e.g., directed-forgetting procedures), is that it allows for a comparative psychology, in which the behavior of humans can be compared with that of other animals through the study of similar phenomena. When making comparisons across species, however, one must ensure both that procedural artifacts are not responsible for spurious similarities and that the task demands are comparable. The study of directed forgetting in animals may now be at a point at which active (controlled) memory processes in animals can be compared with those implicated in human memory.

\section{REFERENCES}

BJORK, R. A. (1972). Theoretical implications of directed forgetting. In A. W. Melton \& E. Martin (Eds.), Coding processes in human memory (pp. 217-235). New York: Winston \& Wiley.

BLougH, D. S. (1959). Delayed matching in the pigeon. Journal of the Experimental Analysis of Behavior, 2, 151-160.

EPSTEIN, W. (1972). Mechanisms of directed forgetting. In G. H. Bower (Ed.), The psychology of learning and motivation (Vol. 6, pp. 147191). New York: Academic Press.

Grant, D. S. (1981). Stimulus control of information processing in pigeon short-term memory. Learning \& Motivation, 12, 19-39.

GRANT, D. S. (1982), Stimulus control of information processing in rat short-term memory. Journal of Experimental Psychology: Animal Behavior Processes, 8, 154-164.

GRANT, D. S. (1984). Directed forgetting and intratrial interference in pigeon delayed matching. Canadian Journal of Psychology, 38, 166-177.

GranT, D. S. (1986). Establishing a forget cue in pigeons using the intratrial interference procedure. Animal Learning \& Behavior, 14, 267-275.

Grant, D. S. (1989). Use of an ambiguous sample procedure to establish a cue to forget in pigeons. Journal of the Experimental Analysis of Behavior, 52, 325-334.

Grant, D. S., \& MacDonald, S. E. (1990). An evaluation of the role of dual coding in mediating the effect of incorrectly cuing the comparison dimension in delayed matching in pigeons. Animal Learning \& Behavior, 18, 151-156.

KENDRICK, D. F., \& RILLING, M. E. (1986). AIM: A theory of active and inactive memory. In D, F. Kendrick, M. E. Rilling, \& M. R. Denny (Eds.), Theories of animal memory (pp. 129-152). Hillsdale, NJ: Erlbaum.

Kendrick, D. F., Rulling, M. E., \& Stonebraker, T. B. (1981). Stimulus control of delayed matching in pigeons: Directed forgetting. Journal of the Experimental Analysis of Behavior, 36, 241-251.

MAKI, W. S., \& HEgVIK, D. K. (1980). Directed forgetting in pigeons. Animal Learning \& Behavior, 8, 567-574.

MAKI, W. S., OLsON, D., \& REGo, S. (1981). Directed forgetting in pigeons: Analysis of cue functions. Animal Learning \& Behavior, 9 , 189-195.

Roberts, W. A., Mazmanian, D. S., \& Kraemer, P. J. (1984). Directed forgetting in monkeys. Animal Learning \& Behavior, 12, 29-40.

ROPER, K. L., \& ZENTALL, T. R. (1993). Directed forgetting in animals. Psychological Bulletin, 113, 513-532.

SaNTI, A., \& SaVICH, J. (1985). Directed forgetting effects in pigeons: Remember cues initiate tehearsal. Animal Learning \& Behavior, 13, 365-369.

Zentall, T. R., Roper, K. L., \& Sherburne, L. M. (1995). Directed forgetting in pigeons can be attributed to the absence of reinforcement on forget trials during training rather than to the absence of a discrimination. Journal of the Experimental Analysis of Behavior, 63, 127-137.

(Manuscript received May 6, 1994; revision accepted for publication October 9, 1994.) 